\title{
Palliative Short-course Radiotherapy in Advanced Pelvic Cancer: A Phase II Study (SHARON Project)
}

\author{
ELEONORA FARINA ${ }^{1}$, GABRIELLA MACCHIA ${ }^{2}$, GIAMBATTISTA SIEPE ${ }^{3}$, \\ ALICE ZAMAGNI $^{3}$, MILLY BUWENGE ${ }^{3}$, ERICA SCIROCCO ${ }^{3}$, FRANCESCO CELLINI $^{4}$, \\ BINIYAM T. DERESSA ${ }^{5}$, WONDEMAGEGNEHU TIGENEH ${ }^{5}$, KAMAL A.F.M. UDDIN ${ }^{6}$, \\ MOSTAFÀ AZIZ SUMON ${ }^{7}$, LUCIANA CARAVATTA $^{8}$, DOMENICO GENOVESI $^{8}$, FLORA ANNA MAURO $^{1}$, \\ SILVIA CAMMELLI ${ }^{3}$, SAVINO CILLA $^{9}$, ALESSIO G. MORGANTI ${ }^{3}$ and FRANCESCO DEODATO ${ }^{2}$ \\ ${ }^{1}$ Radiation Oncology Unit, Maria Cecilia Hospital, GVM Care \& Research, Cotignola, Italy; \\ ${ }^{2}$ Radiotherapy Unit, Department of Oncology, Giovanni Paolo II Foundation, \\ Catholic Sacred Heart University, Campobasso, Italy; \\ ${ }^{3}$ Radiation Oncology Center, Department of Experimental, Diagnostic and Specialty Medicine - \\ DIMES, University of Bologna, S. Orsola-Malpighi Hospital, Bologna, Italy; \\ ${ }^{4}$ Department of Radiotherapy, A. Gemelli Hospital, Catholic University, Rome, Italy; \\ ${ }^{5}$ Radiotherapy Department, Tikur Anbessa Specialized Hospital, Addis Ababa, Ethiopia; \\ ${ }^{6}$ Department of Radiation Oncology, United Hospital Limited, Dhaka, Bangladesh; \\ ${ }^{7}$ Clinical Oncology, Kurmitola General Hospital, Dhaka, Bangladesh; \\ ${ }^{8}$ Department of Radiation Oncology, SS. Annunziata Hospital, G. D'Annunzio University of Chieti, Chieti, Italy; \\ ${ }^{9}$ Medical Physics Unit, Giovanni Paolo II Foundation, Catholic Sacred Heart University, Campobasso, Italy
}

\begin{abstract}
Aim: To define safety and efficacy of a palliative, short-course accelerated radiation therapy for symptomatic locally advanced primary pelvic cancer. Materials and Methods: A phase II trial was planned based on the minimax Simon's twostage design. A total of 18 Gy in $4.5 \mathrm{~Gy} /$ fraction administered twice a day was delivered (SHARON). Pain and quality of life were recorded according to the Visual Analogue self-assessment and the cancer linear analog scales (CLAS), respectively. Results: Twenty-five patients were enrolled in the study. The most frequent baseline symptoms were pain (48\%), bleeding (40\%), bleeding/pain (8\%), and intestinal sub-occlusion (4\%). The overall palliative response rate was $96.0 \%$, with a median palliative duration of 6 months. An improvement of quality-oflife indices (well-being, fatigue, and ability to perform daily activities) was noted in $64.0 \%, 36.0 \%$, and $48.0 \%$ of patients, respectively. Conclusion: The SHARON regimen was well
\end{abstract}

This article is freely accessible online.

Correspondence to: Eleonora Farina, MD, Radiation Oncology Unit, Maria Cecilia Hospital, GVM Care \& Research, Via Corriera 1, 48033 Cotignola (RA), Italy. Tel: +39 0545217167, Fax: +39 0545217160, e-mail: radiotherapy.ef@gmail.com

Key Words: Pelvic cancer, radiotherapy, palliative care, pain, quality of life, phase II. tolerated and effective in the palliative treatment of patients with locally advanced pelvic cancer. Based on these results, a multicentric prospective phase III trial is ongoing to compare this regimen with traditional 2-week radiotherapy treatment.

Symptoms such as pain, bleeding, intestinal/urinary occlusion, nausea, and vomiting can deeply affect the quality of life (QoL) of patients with locally advanced pelvic cancers (1-5). Moreover, for symptomatic patients with metastatic disease or severe comorbidities, radical treatments are generally contraindicated. Palliative radiotherapy (RT) can be an effective option to control symptoms and consequently improve patient QoL (6).

Short-course RT regimens have several advantages in the palliative setting: i) Reduced discomfort for patients, ii) reduced delay of systemic treatment when indicated, iii) reduced delay until hospice admission, and iv) reduced costs for the health system. These advantages are particularly interesting in lesser resourced settings with long waiting lists for RT (7).

Our group previously reported the results of hypofractionated-accelerated RT (delivered in four fractions on 2 consecutive days) in different palliative settings: complicated bone metastases, brain metastases, head and neck cancer, thoracic tumors, and elderly patients (8-13). The treatment was well tolerated and effective in terms of symptom relief. A potential risk while using a hypo-fractionated regimen is the development of long-term radiation-related side-effects 
Table I. Tumor sites and histological types.

\begin{tabular}{lccc}
\hline Tumor site & Histological type & Number of patients $(\mathrm{n}=25)$ & $\%$ \\
\hline Endometrium & Adenocarcinoma & 5 & 20 \\
Colon & Adenocarcinoma & 5 & 20 \\
Prostate & Adenocarcinoma & 3 & 12 \\
Bladder & Urothelial & 3 & 12 \\
Ovary & Total & 3 & 12 \\
& Adenocarcinoma & 1 & 4 \\
& Serous carcinoma & 1 & 4 \\
Vulva & Endometrioid carcinoma & 2 & 8 \\
Rectum & Melanoma & 2 & 8 \\
Synchronous: Bladder + prostate & Adenocarcinoma & 2 & 8 \\
\hline
\end{tabular}

in adjacent radiosensitive organs at risk (14). However, the delivery of two fractions per day can reduce this risk due to the lower fraction size (15).

We previously tested this RT regimen in a phase I doseescalation study on advanced pelvic malignancy and defined the 18 Gy dose level as the maximum-tolerated dose (16). Therefore, the aim of this prospective phase II trial was to assess the efficacy of this protocol in the palliative treatment of advanced symptomatic pelvic malignancies.

\section{Patients and Methods}

Eligibility criteria. Patients with histologically proven locally advanced symptomatic pelvic cancer were enrolled. They were excluded from radical RT due to disease stage or presence of multiple comorbidities or poor Eastern Cooperative Oncology Group performance status (ECOG PS). Other eligibility criteria were: age $\geq 18$ years, ECOG PS $\leq 3$, and no prior RT to the same site. Patients undergoing systemic therapy were enrolled after a 10 day-interval from their last chemotherapy administration. All patients underwent a first evaluation with clinical history, physical examination, blood tests, and pelvic computed tomographic (CT) scan. Information on symptoms, ECOG PS, and QoL were registered at baseline and at each follow-up. Pain was measured on the validated Visual Analogue self-assessment Scale (VAS) (17). Pain intensity and use of analgesics were also recorded according to the International Atomic Energy Agency scale (Pain and Drug scores) (18). The QoL indices were evaluated using the Cancer Linear Analog Scale (CLAS) 1, CLAS2 and CLAS3 visual analog scales for well-being, fatigue, and ability to perform daily activities, respectively. The reliability of these QoL indices have been validated and published (19).

Study design and end-points. The sample size was defined on the minimax Simon's two-stage design (20). This statistical design was to test the null hypothesis that the symptomatic response rate would improve from 10.0 to $30.0 \%$ (with an $\alpha$ and $\beta$ error of 0.05 and 0.2 , respectively) without and with RT, respectively. Based on this design, the enrolment of 15 patients was planned in the first stage of the trial. In the case of $\leq 1$ symptomatic response recorded in the first 15 patients, the closure of the study was planned and the tested regimen was to be considered ineffective. If two symptomatic responses were recorded in the first 15 patients, continuation of the study was planned with further enrollment of 10 additional patients. With $\leq 5 / 25$ symptomatic responses after RT, the treatment would be considered ineffective.

$R T$ planning and delivery. All enrolled patients underwent a CT-scan simulation in supine position. Simulation CT images were taken in $5-\mathrm{mm}$ increments over the region of interest. The gross tumor volume (GTV) was defined as the macroscopic lesion. The clinical target volume (CTV) was defined as the GTV plus $1 \mathrm{~cm}$ isotropic margin. The planning target volume (PTV) was the CTV with $1 \mathrm{~cm}$ isotropic margin. Three-dimensional conformal RT was planned using the Oncentra MasterPlan (Nucletron B.V., Veenendaal, the Netherlands) treatment planning system. A four-field 'box' RT technique was employed in all patients (Figures $1 \mathrm{~A}$ and B). A total of $18 \mathrm{~Gy}$ were delivered in $4.5 \mathrm{~Gy} /$ fraction twice a day with an interval of at least 8 hours between fractions to allow normal tissue repair. Using an $\alpha / \beta$ ratio of 3 , the corresponding equivalent dose in 2 Gy fractions (EQD2) was $27.8 \mathrm{~Gy}$ (21). The dose was specified according to the International Commission on Radiation Units and Measurements Report 62 (22). Dose-volume histograms were calculated for PTV and organs at risk. The treatment was delivered by an Elekta Precise Linac (Elekta AB, Stockholm, Sweden) equipped with standard multi-leaf collimators. During treatment planning and delivery, the medical and physics staff performed two independent checks for quality assurance (23). Treatment reproducibility was checked before any fraction using an electronic portal imaging device (24).

Follow-up evaluation. Fifteen days after RT, all patients underwent a first evaluation. Afterwards, patients were followed at 2-month intervals. Evaluation consisted of clinical history, physical examination, and blood test. Acute and late radiation-related toxicities were scored according to the Radiation Therapy Oncology Group (RTOG) and the European Organisation for Research and Treatment of Cancer (EORTC) - RTOG toxicity scales, respectively (25). Indices related to symptom relief, pain, drug score and QoL were recorded. Bleeding and intestinal sub-occlusion were considered to be completely palliated when the patient required no further medication to control symptoms. Complete pain relief was defined as a VAS 

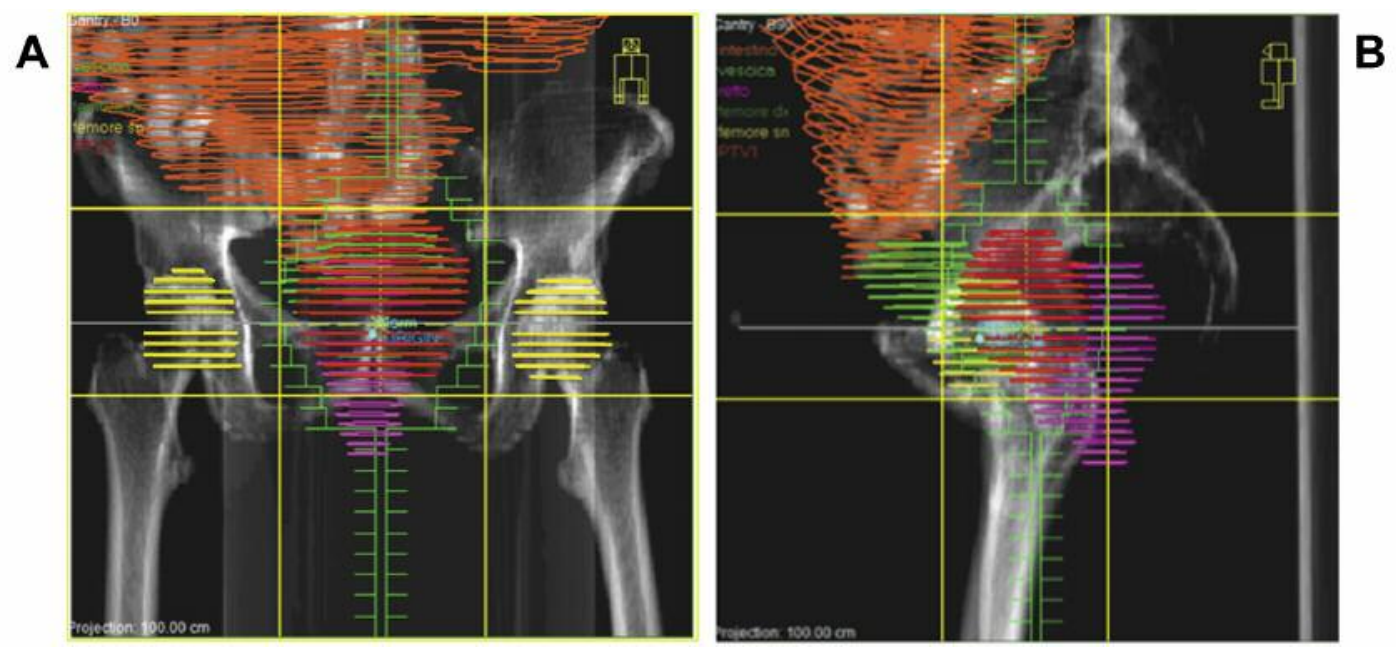

Figure 1. Field arrangement (A: anterior-posterior field; B: lateral field) in a patient with bleeding cervical cancer recurrence showing the planning target volume (red) coverage and sparing of organs at risk (orange: small bowel; purple: rectum; clear green: bladder; yellow: femoral heads).
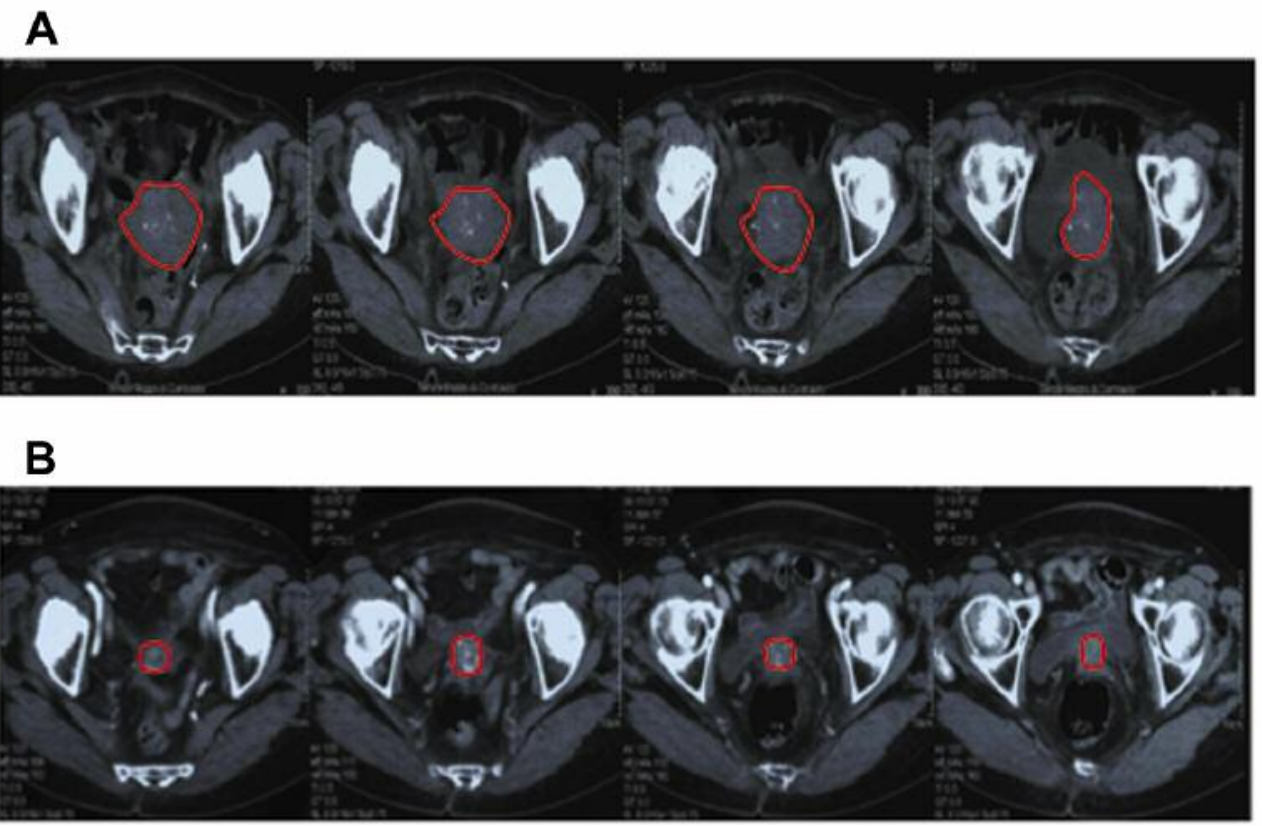

Figure 2. Gross tumor volume (highlighted by the red line) before (A) and 1 month after (B) SHARON treatment in the same patient of Figure 1. The patient showed a partial tumor response but complete response regarding bleeding.

score of 0 . A reduction without complete resolution of symptom severity, or a decrease in pain and drug score were considered to be a partial response. In addition, information about ECOG PS and QoL obtained after radiotherapy was compared to that at baseline and any difference was graded as improved, stable, or worse.

\section{Results}

Patient characteristics. A total of 25 patients (male/female: 13/12; median age: 72 years, range $=43-89$ years; ECOG PS
1/2/3: 10/10/5 patients, respectively) were enrolled in this phase II trial. The overall primary sites were endometrium in $20 \%$, sigmoid bowel in $20 \%$, prostate in $12 \%$, bladder in $12 \%$, ovary in $12 \%$, vulva in $8 \%$, rectum in $8 \%$, and synchronous cancer in $8 \%$. Tumor sites and histopathology of the primary tumors are detailed in Table I. Overall, the most frequent baseline symptoms were pain (48\%), bleeding alone $(40 \%)$, bleeding/pain $(8 \%)$, and bowel sub-occlusion $(4 \%)$. (prostate and bladder). 
Response. In the first stage of the trial's design, 15 patients were enrolled and treated. Four patients (26.6\%) experienced complete symptom remission, $10(66.7 \%)$ a partial response, and there was no change in one patient $(6.7 \%)$. Thus, the palliative response rate (complete plus partial symptom remission) in the first stage was $93.3 \%$. Based on these results, the second stage of the trial commenced to reach the planned total number of 25 patients.

The overall palliative response rate (complete plus partial symptom remission) for the whole patients' cohort was $96.0 \%$ (95\% confidence intervaI=78.9->99.9\%). Moreover, eight patients (32\%) experienced complete symptom remission, 16 patients $(64 \%)$ reported a partial response, and one (4\%) had no change. Median palliation duration was 6 months. The overall response rate for pain was $92.9 \%$ (95\% $\mathrm{CI}=66.5->99.9 \%)$. The pre- and post-treatment mean VAS was 5.6 and 2.7 , respectively $(p<0.001)$. For pain relief alone or associated with bleeding, two out of 14 patients (14.3\%) had complete symptom resolution (VAS $=0), 11$ out of 14 (78.6\%) had partial symptom resolution (median $\Delta$ VAS $=48.5 \% ; \Delta$ VAS range $=16.7-83.3 \%$ ), one out of 14 (7.1\%) had no change, and none experienced increased pain. Seven patients (58.3\%) with bleeding (alone or associated with pain) reported complete symptom resolution, five (41.7\%) had partial resolution, and none experienced no change or symptom progression. The only patient with intestinal sub-occlusion showed a partial symptomatic relief.

At the first follow-up, 13 patients (52.0\%) had improved ECOG PS, $11(44.0 \%)$ were stable, and one $(4.0 \%)$ had experienced deterioration. An improvement of CLAS1, CLAS2 and CLAS3 indices was noted in $64.0 \%, 36.0 \%$, and $48.0 \%$ of patients, respectively, while stability of their values was recorded in $12.0 \%, 32.0 \%$, and $20.0 \%$, and deterioration in $24.0 \%, 32.0 \%$, and $32.0 \%$, respectively (Figure 2A and B).

Toxicity. Acute grade 1 skin toxicity was recorded in two patients (8.0\%). Grade 1 and 2 acute gastrointestinal and genitourinary toxicities were reported by six $(24.0 \%)$ and seven patients (28.0\%), respectively. No patient had grade 3 or more toxicity. With a median follow-up of 6 months (range $=2-26$ months), no patient experienced late toxicity.

\section{Discussion}

A phase II study was planned to evaluate the symptomatic response rate of a short-course palliative RT treatment in advanced pelvic cancer. To our knowledge, this is the first trial testing the palliative efficacy of a hypo-fractionated accelerated RT regimen with the delivery of a total dose of $18 \mathrm{~Gy}$ in this setting. The study showed this regimen achieved an overall palliative response rate of $96.0 \%(95 \%$ $\mathrm{CI}=78.9->99.9 \%)$ with a median duration of palliation of 6 months.
The main limitations of the study include the small sample size and the short follow-up time, not allowing a definitive assessment of late toxicity.

Our results compare favorably with other studies on palliative RT in advanced pelvic cancer. The first study to propose the $2 \times 2$ fractionation in the palliation of advanced pelvic cancer was the RTOG 8502 trial in the late 1980s (26). The study evaluated a regimen of $3.7 \mathrm{~Gy} /$ fraction b.i.d. over 2 days for a total dose of $14.8 \mathrm{~Gy}$, which was eventually repeated three times to a total dose of $44.4 \mathrm{~Gy}$ (26). The study showed an overall palliative response rate of $32 \%$ ( $45 \%$ for patients completing all 3 cycles) in comparison with a rate of $96.0 \%$ obtained with our protocol (26). The reason for this difference may be due to the higher dose delivered in our trial (18 Gy versus $14.8 \mathrm{~Gy}$ ).

Another trial on locally advanced cervical cancer was based on the delivery of $10 \mathrm{~Gy}$ in a single fraction monthly up to a maximum of three fractions. The overall response for bleeding and pain were $100 \%$ and $33 \%$, respectively, versus $100 \%$ and $92.9 \%$ recorded in our study, respectively (27). Again, the differences between the results of the two studies in terms of pain relief may be related to the different doses delivered. However, it should be noted that the response on bleeding seems to be independent of the RT protocol used.

In a study on palliative pelvic RT (30-39 Gy in 10-13 fractions) for symptomatic incurable prostate cancer, the overall symptomatic response rate was $62 \%$ again lower compared to our results (28). This difference may be due to the different symptomatic profile of patients with prostate cancer who present with lower urinary tract symptoms as the most frequent tumor-related complication.

In another study, the delivery of $25 \mathrm{~Gy}$ in five fractions (1 fraction/day) in symptomatic rectal cancer treated for palliation led to similar response rates (100\% for bleeding, and $87.5 \%$ for pain) but with a slightly longer treatment time (29).

In conclusion, our results suggest that the palliative regimen used in this study, based on the delivery of hypo-fractionated accelerated RT, is well tolerated and able to achieve a high rate of symptomatic responses. Therefore, it might be considered a treatment option particularly for patients for whom a longer treatment time might be a source of discomfort.

Moreover, our results justify further studies which might be addressed to: i) Evaluating the effect of higher doses delivered with more advanced treatment techniques (intensity-modulated RT, volumetric-modulated arc therapy); ii) evaluating the feasibility and tolerability of this regimen delivered with less advanced techniques (2D-RT) in lesser resourced settings, where treatment brevity might be particularly useful; iii) comparing tolerability and efficacy of this protocol with more traditional RT regimens.

For these reasons, a multicentric prospective phase III trial coordinated by our center is ongoing to compare the SHARON regimen to a more conventional one (30 Gy in 10 fractions). 


\section{Conflicts of Interest}

All Authors declare they have no financial and personal relationships with other people or organizations that inappropriately influence (bias) their work.

\section{Funding}

This research did not receive any specific grant from funding agencies in the public, commercial, or not-for-profit sector.

\section{Ethical Considerations}

The study was approved by the local Ethics Committee (n. UCSCCB-2009/32). All procedures were approved by the Institutional Ethic Committee and were in accordance with the Helsinki Declaration as revised in 2008. All patients provided a written informed consent before study entry.

\section{Authors' Contributions}

All Authors have participated sufficiently in the work and contributed to the conception and design of the study. G. Macchia, F. Deodato and A.G. Morganti participated in the acquisition of data. A.G. Morganti, M. Buwenge and E. Farina participated in statistical data analysis. All Authors participated in the interpretation of data, drafting the article, and revising it critically. All Authors contributed to the final approval of the manuscript.

\section{References}

1 Rigor BM Sr.: Pelvic cancer pain. J Surg Oncol 75(4): 280-300, 2000. PMID: 11135274.

2 Lim AWW, Forbes LJL, Rosenthal AN, Raju KS and Ramirez A-J: Measuring the nature and duration of symptoms of cervical cancer in young women: Developing an interview-based approach. BMC Women's Health 13: 45, 2013. PMID: 24219028. DOI: $10.1186 / 1472-6874-13-45$

3 Low EL, Simon AE, Waller J, Wardle J and Menon U: Experience of symptoms indicative of gynaecological cancers in UK women. Br J Cancer 109(4): 882-887, 2013. PMID: 23880819. DOI: $10.1038 /$ bjc.2013.412

4 Astin M, Griffin T, Neal RD, Rose P and Hamilton W: The diagnostic value of symptoms for colorectal cancer in primary care: A systematic review. Br J Gen Pract 61(586): e231-e243, 2011. PMID: 21619747. DOI: 10.3399/bjgp11X572427

5 DeGeorge KC, Holt HR and Hodges SC: Bladder cancer: Diagnosis and treatment. Am Fam Physician 96(8): 507-514, 2017. PMID: 29094888.

6 Lutz S, Korytko T, Nguyen J, Khan L, Chow E and Corn B: Palliative radiotherapy: When is it worth it and when is it not? Cancer J 16(5): 473-482, 2010. PMID: 20890143. DOI: 10.1097/ PPO.0b013e3181f28b4d

7 Rodin D, Grover S, Elmore SN, Knaul FM, Atun R, Caulley L, Herrera CA, Jones JA, Price AJ, Munshi A, Gandhi AK, Shah C and Gospodarowicz M: The power of integration: Radiotherapy and global palliative care. Ann Palliat Med 5(3): 209-217, 2016. PMID: 27481320. DOI: 10.21037/apm.2016.06.03
8 Capuccini J, Macchia G, Farina E, Buwenge M, Genovesi D, Caravatta L, Nguyen NP, Cammelli S, Cilla S, Wondemagegnhu T, Uddin AFMK, Aziz Sumon M, Cellini F, Valentini V, Deodato $F$ and Morganti AG: Short-course regimen of palliative radiotherapy in complicated bone metastases: A phase I-II study (SHARON Project). Clin Exp Metastasis 35(7): 605-611, 2018. PMID: 30121938. DOI: 10.1007/s10585-018-9931-9

9 Caravatta L, Deodato F, Ferro M, Macchia G, Massaccesi M, Cilla S, Padula GD, Mignogna S, Tambaro R, Carrozza F, Flocco M, Cantore G, Scapati A, Buwenge M, Sticca G, Valentini V, Cellini $\mathrm{N}$ and Morganti AG: A phase I study of short-course accelerated whole-brain radiation therapy for multiple brain metastases. Int J Radiat Oncol Biol Phys 84(4): e463-8, 2012. PMID: 22909415. DOI: 10.1016/j.jjrobp. 2012.06.023

10 Caravatta L, Deodato F, Ferro M, Macchia G, Massaccesi M, Cilla S, Tambaro R, Mignogna S, Padula GD, Musacchio M, Flocco M, Cantore G, Scapati A, Bogale S, Balducci M, Valentini V, Cellini $\mathrm{N}$ and Morganti AG: Results of a phase II study of Short-course Accelerated Radiation Therapy (SHARON) for multiple brain metastases. Am J Clin Oncol 38(4): 395-400, 2015. PMID: 26214084. DOI: 10.1097/ COC.0b013e3182a0e826

11 Farina E, Capuccini J, Macchia G, Caravatta L, Nguyen NP, Cammelli S, Farioli A, Zanirato Rambaldi G, Cilla S, Wondemagegnhu T, Uddin AFMK, Sumon MA, Genovesi D, Buwenge M, Cellini F, Valentini V, Deodato F and Morganti AG: Phase I-II study of Short-course Accelerated Radiotherapy (SHARON) for palliation in head and neck cancer. Anticancer Res 38(4): 2409-2414, 2018. PMID: 29599369. DOI: 10.21873/ anticanres.12491

12 Farina E, Macchia G, Buwenge M, Siepe G, Zamagni A, Cammelli S, Cilla S, Wondemagegnhu T, Woldemariam AA, Uddin AFMK, Sumon MA, Cellini F, Deodato F and Morganti AG: Radiotherapy in palliation of thoracic tumors: A phase I-II study (SHARON project). Clin Exp Metastasis 35(8): 739-746, 2018. PMID: 30298381. DOI: 10.1007/s10585-018-9942-6

13 Farina E, Capuccini J, Macchia G, Caravatta L, Nguyen NP, Cammelli S, Zanirato Rambaldi G, Cilla S, Wondemagegnhu T, Uddin AFMK, Sumon MA, Genovesi D, Buwenge M, Cellini F, Valentini V, Deodato $F$ and Morganti AG: Short course accelerated radiation therapy (SHARON) in palliative treatment of advanced solid cancer in older patients: a pooled analysis. J Geriatr Oncol 9(4): 359-361, 2018. PMID: 29398455. DOI: 10.1016/j.jgo.2018.01.004

14 Jones B, Dale RG, Finst P and Khaksar SJ: Biological equivalent dose assessment of the consequences of hypofractionated radiotherapy. Int J Radiat Oncol Biol Phys 47(5): 1379-1384, 2000. PMID: 10889393.

15 Muriel VP: The biological basis of fractionation in radiotherapy. Rev Oncol 4: 161-166, 2002. DOI: 10.1007/BF02732348.

16 Caravatta L, Padula GDA, Macchia G, Ferrandina G, Bonomo P, Deodato F, Massaccesi M, Mignogna S, Tambaro R, Rossi M, Flocco M, Scapati A, Scambia G, Pacelli F, Valentini V, Cellini $\mathrm{N}$ and Morganti AG: SHort course Accelerated RadiatiON therapy (SHARON) in palliative treatment of advanced pelvic malignancies: A phase I study. Int J Radiat Oncol Biol Phys 83(5): e627-631, 2012. PMID: 22580117. DOI: 10.1016/j.ijrobp. 2011.10.081

17 Melzack R: The McGill Pain Questionnaire: Major properties and scoring methods. Pain 1(3): 277-299, 1975. PMID: 1235985.

18 Salazar OM, Sandhu T, da Motta NW, Escutia MA, Lanzós- 
Gonzales E, Mouelle-Sone A, Moscol A, Zaharia M and Zaman $S$ : Fractionated half-body irradiation (HBI) for the rapid palliation of widespread, symptomatic, metastatic bone disease: a randomized phase III trial of the International Atomic Energy Agency (IAEA). Int J Radiat Oncol Biol Phys 50(3): 765-75, 2001. PMID: 11395246.

19 Sutherland HJ, Walker P and Til JE: The development of a method for determining oncology patients' emotional distress using linear analogue scales. Cancer Nurs 11(5): 303-308, 1988. PMID: 3233581.

20 Simon R, Wittes RE and Ellenberg SS: Randomized phase II clinical trials. Cancer Treatment Rep 69(12): 1375-1381, 1985. PMID: 4075313.

21 Withers HR: Biological basis of radiation therapy. In: Principles and Practice of Radiation Oncology. Perez CA and Brady LW (eds.). Philadelphia, Lippincott, pp. 64-69, 1992.

22 International Commission on Radiation Units and Measurements, ICRU report 62: Prescribing, Recording and Reporting Photon Beam Therapy (supplement to ICRU report 50). Bethesda, 1999.

23 Morganti AG, Deodato F, Zizzari S, Cilla S, Digesù C, Macchia G, Panunzi S, De Gaetano A, Piermattei A, Cellini N and Valentini V: Complexity index (COMIX) and not type of treatment predicts undetected errors in radiotherapy planning and delivery. Radiother Oncol 89(3): 320-329, 2008. PMID: 18701176. DOI: 10.1016/j.radonc.2008.07.009

24 Deodato F, Cilla S, Massaccesi M, Macchia G, Ippolito E, Caravatta L, Picardi V, Romanella M, Di Falco C, Bartollino A, Valentini V, Cellini N, De Spirito M, Piermattei A and Morganti AG: Daily on-line setup correction in 3D-conformal radiotherapy: Is it feasible? Tumori 98(4): 441-444, 2012. PMID: 23052159. DOI: 10.1700/1146.12637

25 Cox JD, Stetz J and Pajak TF: Toxicity criteria of the Radiation Therapy Oncology Group (RTOG) and the European Organization for Research and Treatment of Cancer (EORTC). Int J Radiat Oncol Biol Phys 31(5): 1341-1346, 1995. PMID: 7713792. DOI: 10.1016/0360-3016(95)00060-C
26 Spanos W Jr., Guse C, Perez C, Grigsby P, Doggett RLS and Poulter C: Phase II study of multiple daily fractionations in the palliation of advanced pelvic malignancies: preliminary report of RTOG 8502. Int J Radiat Oncol Biol Phys 17(3): 659-661, 1989. PMID: 2476426.

27 Mishra SK, Laskar S, Muckaden MA, Mohindra P, Shrivastava SK and Dinshaw KA: Monthly palliative pelvic radiotherapy in advanced carcinoma of uterine cervix. J Cancer Res Ther 1(4): 208-212, 2005. PMID: 17998655.

28 Cameron MG, Kersten C, Vistad I, van Helvoirt R, Weyde K, Undseth C, Mjaaland I, Skovlund E, Fosså SD and Guren MG: Palliative pelvic radiotherapy for symptomatic incurable prostate cancer - A prospective multicenter study. Radiother Oncol 115(3): 314-320, 2015. PMID: 26091575. DOI: 10.1016/ j.radonc.2015.05.021

29 Picardi V, Deodato F, Guido A, Giaccherini L, Macchia G, Frazzoni L, Farioli A, Cuicchi D, Cilla S, Cellini F, Uddin AF, Gambacorta MA, Buwenge M, Ardizzoni A, Poggioli G, Valentini V, Fuccio L and Morganti AG: Palliative short-course radiation therapy in rectal cancer: A phase 2 study. Int J Radiat Oncol Biol Phys 95(4): 1184-1190, 2016. PMID: 27215449. DOI: $10.1016 /$ j.ijrobp.2016.03.010 